Research Paper

\title{
Circulating tumor cells and microemboli can differentiate malignant and benign pulmonary lesions
}

\author{
Mario Mascalchi ${ }^{1}$; Cristina Maddau ${ }^{2}$; Lapo Sali ${ }^{1}$; Elena Bertelli ${ }^{1}$; Francesca Salvianti ${ }^{3}$; Stefania Zuccherelli1 ${ }^{1}$; \\ Marzia Matucci²; Alessandra Borgheresi ${ }^{1}$; Claudio Raspanti ${ }^{4}$; Monica Lanzetta ${ }^{1}$; Massimo Falchini ${ }^{1}$; Ernesto \\ Mazza4; Alessandra Vella5; Michaela Luconi6; Pamela Pinzani3; Mario Pazzagli ${ }^{3 凶}$ \\ 1. Diagnostic and Interventional Radiology Units, "Mario Serio" Department of Biomedical, Experimental and Clinical Sciences, University of Florence, \\ Florence, Italy; \\ 2. Oncological Prevention Laboratory, Institute for Cancer Research and Prevention (ISPO), Florence, Italy; \\ 3. Clinical Biochemistry Unit, "Mario Serio" Department of Biomedical, Experimental and Clinical Sciences, University of Florence, Florence, Italy; \\ 4. Interventional Radiology Unit, Careggi Hospital, Florence, Italy; \\ 5. Nuclear Medicine Unit, Le Scotte University Hospital, Siena, Italy; \\ 6. Endocrinology Unit, "Mario Serio" Department of Biomedical, Experimental and Clinical Sciences, University of Florence, Florence, Italy. \\ $\triangle$ Corresponding author: Professor Mario Mascalchi: "Mario Serio" Department of Biomedical, Experimental and Clinical Sciences, University of Florence, \\ Viale Morgagni 50, 50134 Florence, Italy. E-mail: mario.mascalchi@unifi.it. \\ (C) Ivyspring International Publisher. This is an open access article distributed under the terms of the Creative Commons Attribution (CC BY-NC) license \\ (https://creativecommons.org/licenses/by-nc/4.0/). See http://ivyspring.com/terms for full terms and conditions.
}

Received: 2016.11.19; Accepted: 2017.02.26; Published: 2017.07.15

\begin{abstract}
The presence of circulating tumor cells (CTC) or microemboli (CTM) in the peripheral blood can theoretically anticipate malignancy of solid lesions in a variety of organs. We aimed to preliminarily assess this capability in patients with pulmonary lesions of suspected malignant nature.

We used a cell-size filtration method (ScreenCell) and cytomorphometric criteria to detect CTC/CTM in a $3 \mathrm{~mL}$ sample of peripheral blood that was taken just before diagnostic percutaneous CT-guided fine needle aspiration (FNA) or core biopsy of the suspicious lung lesion. At least one CTC/CTM was found in 47 of 67 (70\%) patients with final diagnoses of lung malignancy and in none of 8 patients with benign pulmonary nodules. In particular they were detected in 38 (69\%) of 55 primary lung cancers and in $9(75 \%)$ of 12 lung metastases from extra-pulmonary cancers. Sensitivity of CTC/CTM presence for malignancy was $70.1 \%(95 \% \mathrm{Cl}: 56.9-83.1 \%)$, specificity $100 \%$, positive predictive value $100 \%$ and negative predictive value $28.6 \%(95 \% \mathrm{Cl}$ : $11.9-45.3 \%)$. Remarkably, the presence of CTC/CTM anticipated the diagnosis of primary lung cancer in 3 of 5 patients with non-diagnostic or inconclusive results of FNA or core biopsy, whereas CTC/CTM were not observed in 1 patient with sarcoidosis and 1 with amarthocondroma.

These results suggest that presently, due to the low sensitivity, the search of CTC/CTM cannot replace CT guided percutaneous FNA or core biopsy in the diagnostic work-up of patients with suspicious malignant lung lesions. However, the high specificity may as yet indicate a role in cases with non-diagnostic or inconclusive FNA or core biopsy results that warrants to be further investigated.
\end{abstract}

Key words: circulating tumor cells; lung nodule; lung cancer; lung metastases; CT-guided fine needle aspiration.

\section{Introduction}

The term "liquid biopsy" refers to the search and characterization in the peripheral blood of both circulating tumor cells (CTC) or microemboli (CTM) and biomolecules, such as free circulating DNA and
micro-RNA [1]. "Liquid biopsy" is receiving growing attention in lung oncology because it can non-invasively and repeatedly provide valuable information that is crucial for prognosis and treatment 
modulation according to a personalized medicine approach [2-4].

The presence of CTC or CTM can theoretically anticipate malignancy of solid lesions detected in a variety of organs and hence have a diagnostic value. So far, this possibility has been investigated in patients with breast, gastric, pancreatic and prostatic lesions [5-8]. Lung is one special organ as it frequently harbors primary or secondary cancer lesions. The differential diagnosis with a variety of benign lung lesions is a very common clinical problem that is usually addressed by percutaneous or transbronchial fine needle aspiration (FNA) or core biopsy $[9,10]$.

In this study, we aimed to preliminarily determine the predictive value for malignancy of detection of CTC/CTM in patients with single or multiple suspicious pulmonary lesions.

\section{Patients and methods}

\section{Patients selection}

This prospective study recruited 75 consecutive patients undergoing diagnostic percutaneous CT-guided FNA or core biopsy of pulmonary lesions of suspected malignant nature at the Diagnostic and Interventional Radiology Unit of the Careggi University Hospital of Florence, Italy. The study was approved by the Local Ethical Committee (decision May 18, 2015; protocol number BIO 15.009) and each patient provided a written consent to participate to the study.

Eligible patients were those with single or multiple peripheral lung nodules or masses amenable of percutaneous CT-guided FNA or core biopsy for diagnostic purposes. We excluded patients who refused consent to blood sampling, those with a history in the last 5 years of pulmonary and extra-pulmonary malignancy or who had received chemo or radiation therapy. Moreover, to avoid the possibility of CTC delivery in the bloodstream as a result of tumor manipulation $[11,12]$ we also excluded patients who had undergone diagnostic transbronchial FNA or core biopsy within 7 days before the percutaneous procedure.

\section{Blood sample collection}

In each patient, $7 \mathrm{~mL}$ of blood were collected in one $10 \mathrm{~mL}$ EDTA tube just before CT-guided FNA or core biopsy through the antecubital vein access routinely prepared before the procedure.

\section{Percutaneous CT-guided FNA and core biopsy}

Percutaneous CT-guided FNA biopsy of the target lung lesion was performed with a 25-23 gauge needle by one of four interventional radiologists on a
40 rows of detectors Fluoro-CT scanner (Sensation Open, Siemens, Erlangen, Germany) dedicated to interventional procedures. $\mathrm{FN}$ aspirates were processed and analyzed by one of the two expert cytopathologists with Rapid On Site Examination (ROSE) $[9,13]$ and cytological diagnosis was made according to the 2015 lung cancer classification [10]. Immunocytochemistry on FNA material for primary lung cancer was performed in 34 patients in whom typical morphological patterns for adenocarcinoma, squamous cell carcinoma or neuroendocrine tumors were not present. In particular on the FNA material, but not on the isolated CTC/CTM (see below), a limited panel of TTF1, P63 and CD56 markers to enable diagnosis of lung adenocarcinoma, squamous cell carcinoma or small cell carcinoma, respectively, was used. Moreover, in 5 patients we performed immunocytochemistry on FNA material to differentiate metastasis of colorectal cancer or breast cancer from primary lung cancer. We used a panel with TTF1, cytokeratin 7 and cytokeratin 20, estrogen and progesterone receptor for such a purpose.

In 20 cases, FNA was supplemented by core biopsy performed with a 20-16 gauge needle and the latter was evaluated with routine histological procedures.

\section{CTC analysis}

Blood samples were processed within 3 hours after collection and CTC analysis was performed according to a standardized procedure reported in a previous study dealing with a different series of patients, selected because of advanced lung cancer [14]. For each patient, we prepared 2 filters corresponding to a $6 \mathrm{~mL}$ volume of blood. The second filter served as backup in case of technical failures due to filter contamination or incomplete filtration and was stained on demand.

\section{Isolation}

Blood was filtered by the ScreenCell Cyto filtration devices according to the procedure previously described $[14,15]$ that allows isolation by cell size and does not involve any biological characterization of the putative CTC. Before filtration and in order to lyse red blood cells, $3 \mathrm{~mL}$ of the blood sample were diluted in $4 \mathrm{~mL}$ of the proprietary dilution buffer, according to the manufacturer's instructions (ScreenCell FC dilution buffer; ScreenCell). After blood filtration, an additional 1.6 $\mathrm{mL}$ of PBS was filtered to remove red blood cell debris. Filtration was usually completed within approximately 3 minutes. The filter was then disassembled from the filtration module and allowed to air-dry. 


\section{Identification}

After an average of 10 days since FNA or core biopsy (range 7-21 days), the filters were evaluated in consensus by the same two expert cytopathologists (C.M. and M.Mat.) who had performed ROSE. They were blind to both the identity of the patient and the FNA or core biopsy results.

Cytological assessment was conducted directly on the filter. The track-etched filters were stained with hematoxylin solution $S$ (Merck KGaA), applied to the membrane for 2 minutes, and with Shandon eosin $Y$ aqueous (Thermo Electron Corporation, Thermo Fisher Scientific Inc) for 30 seconds. For microscopic observation, the ScreenCell Cyto filter was placed on a standard microscopy glass slide, and a 7-mm circular cover slip (Menzel-Glaser) was laid on the filter with the appropriate mounting medium.

CTC were identified among the cells retained on filter according to the presence of at least four of the following cytomorphological criteria: nucleus size $\geq 16$ $\mu \mathrm{m}$, anisonucleosis (ratio $>0.5)$, high $(>2: 1)$ nuclear/cytoplasmic ratio, irregular nuclei and presence of three-dimensional sheets [14-16]. CTM were defined as clusters formed by $\geq 3$ CTC [17].

\section{Diagnostic work-up and staging}

Final diagnoses were based on the results of FNA or core biopsy. In case of non-diagnostic (due to insufficient material) or inconclusive (doubtful features) results of CT-guided FNA or core-biopsy, diagnosis was based on clinical, laboratory, CT follow-up data or surgical pathology results.

The clinical or pathologic stage of ultimately diagnosed lung cancers was determined according to the 2009 Staging System [18].

\section{Statistical analysis}

A basic statistical evaluation was performed with computation of sensitivity, specificity, positive predictive value (PPV) and negative predictive value (NPV) of CTC/CTM presence with regard to the final diagnosis of malignant or benign pulmonary lesion.

\section{Results}

The demographic data, the results of percutaneous CT-guided FNA or core biopsy, of the search of CTC/CTM, and the final diagnoses of the target lung lesions in the 75 patients are detailed in Table 1. Final diagnosis of primary lung cancer was made in 55 (23 stage I, 6 stage II, 9 stage III and 17 stage IV) and of benign lung nodule in 8 patients. In particular, FNA or core biopsy demonstrated primary lung cancer in 55 patients (38 adenocarcinomas, 12 squamous cell carcinomas, 1 not otherwise specified non small cell lung cancer, 1 small cell lung cancer, 2 carcinoid tumors, 1 neuroendocrine tumor with malignant features), secondary lesions (1 urothelial, 3 head and neck, 4 intestinal, 2 breast, 1 pancreas, 1 neuroendocrine) in 12 patients and benign lesions in 8 patients (3 amarthocondroma, 3 inflammation, 1 abscess, 1 sarcoidosis). In particular, the percutaneous CT guided procedure was non-diagnostic in 4 patients and inconclusive in 1 (overall 5/75=6.7\%). Surgical pathology and follow-up data established diagnosis of primary lung cancer (adenocarcinoma) in 3 cases and of sarcoidosis and amarthocondroma in 1 case each.

Overall, in 18 patients it was necessary to stain and evaluate the second filter due to technical problems of incomplete filtration, presence of white blood elements and Candida albicans contamination that obscured possible CTC presence.

Figure 1 graphically shows the results of CTC/CTM search and of FNA or core biopsy in the 75 patients. At least one CTC/CTM in $3 \mathrm{~mL}$ of blood was found in 47 of 67 (70\%) patients with final diagnoses of lung malignancy and in none of 8 patients with benign pulmonary nodules. In particular they were detected in 38 (69\%) of 55 primary lung cancers and in $9(75 \%)$ of 12 lung metastases from extra-pulmonary cancers.

Table 1. CTC/CTM and diagnoses in patients undergoing percutaneous CT-guided FNA or core biopsy.

\begin{tabular}{|c|c|c|c|c|c|c|c|}
\hline N. & $\begin{array}{l}\text { Patient Age, } \\
\text { Sex }\end{array}$ & FNAB & Core Biopsy & CTC & CTM & Stage & Final diagnosis \\
\hline 1 & $81, \mathrm{M}$ & Squamous cell ca & - & 3 & 1 & IIa & Squamous cell ca \\
\hline 2 & $69, \mathrm{~F}$ & Adenocarcinoma & - & 2 & 3 & pT1aN2IIIa & Adenocarcinoma \\
\hline 3 & $64, \mathrm{M}$ & Squamous cell ca & - & 12 & & Ia & Squamous cell ca \\
\hline 4 & $79, \mathrm{M}$ & Adenocarcinoma & - & 2 & 4 & IV & Adenocarcinoma \\
\hline 5 & $79, \mathrm{~F}$ & Adenocarcinoma & - & 8 & 2 & IV & Adenocarcinoma \\
\hline 6 & $65, \mathrm{~F}$ & Mtx (Squamous cell ca) & - & 1 & 5 & - & Mtx (Squamous cell ca Head \& Neck) \\
\hline 7 & $69, \mathrm{~F}$ & Inflammatory cells & Inflammation & - & - & - & Inflammation \\
\hline 8 & $74, \mathrm{~F}$ & Adenocarcinoma & - & 2 & 1 & IV & Adenocarcinoma \\
\hline 9 & $66, \mathrm{M}$ & Adenocarcinoma & Adenocarcinoma & 8 & 2 & IV & Adenocarcinoma \\
\hline 10 & $87, \mathrm{M}$ & Mtx (Urothelial ca) & - & - & - & - & Mtx (Urothelial ca) \\
\hline 11 & $74, \mathrm{M}$ & Squamous cell ca & - & 6 & 11 & IIIa & Squamous cell ca \\
\hline 12 & $75, \mathrm{M}$ & Adenocarcinoma & - & 6 & 23 & IV & Adenocarcinoma \\
\hline
\end{tabular}




\begin{tabular}{|c|c|c|c|c|c|c|c|}
\hline 13 & $67, \mathrm{M}$ & Adenocarcinoma & - & 6 & 2 & pT1bN0Ia & Adenocarcinoma \\
\hline 14 & $83, \mathrm{M}$ & Mtx (Squamous cell ca) & - & 6 & 4 & - & Mtx (Squamous cell ca Head \& Neck) \\
\hline 15 & $74, \mathrm{M}$ & Inflammatory cells & Inflammation & - & - & - & Inflammation \\
\hline 16 & $79, \mathrm{~F}$ & Adenocarcinoma & - & 4 & 3 & IIa & Adenocarcinoma \\
\hline 17 & $74, \mathrm{M}$ & Amarthocondroma & - & - & - & - & Amarthocondroma \\
\hline 18 & $79, \mathrm{~F}$ & Adenocarcinoma & - & 9 & 4 & IIIa & Adenocarcinoma \\
\hline 19 & $82, \mathrm{~F}$ & Adenocarcinoma & Insufficient material & - & 1 & $\mathrm{IIIb}$ & Adenocarcinoma \\
\hline 20 & $81, \mathrm{M}$ & Adenocarcinoma & - & 8 & 18 & IV & Adenocarcinoma \\
\hline 21 & $64, \mathrm{~F}$ & Mtx (Squamous cell ca) & - & - & - & - & Mtx (Squamous cell ca Larynx) \\
\hline 22 & $73, \mathrm{M}$ & Insufficient material & - & - & - & - & Sarcoidosis \\
\hline 23 & $75, \mathrm{M}$ & Squamous cell ca & - & 2 & 5 & pT1aN0Ia & Squamous cell ca \\
\hline 24 & $71, \mathrm{M}$ & Adenocarcinoma & - & 5 & 9 & IV & Adenocarcinoma \\
\hline 25 & $72, \mathrm{M}$ & Adenocarcinoma & - & 8 & 2 & pT1bN0Ia & Adenocarcinoma \\
\hline 26 & $65, \mathrm{M}$ & Adenocarcinoma & - & - & 2 & pT1aN0Ia & Adenocarcinoma \\
\hline 27 & $75, \mathrm{M}$ & Squamous cell ca & - & 2 & - & IIIa & Squamous cell ca \\
\hline 28 & $67, \mathrm{M}$ & NSCLC NOS & - & - & 3 & IV & NSCLC NOS \\
\hline 29 & $57, \mathrm{~F}$ & Adenocarcinoma & - & 5 & 1 & IIIa & Adenocarcinoma \\
\hline 30 & $68, \mathrm{~F}$ & Adenocarcinoma & - & 4 & 3 & pT1aN0Ia & Adenocarcinoma \\
\hline 31 & $78, \mathrm{M}$ & Mtx (Intestinal Adenocarcinoma) & - & - & - & - & $\begin{array}{l}\text { Mtx (Colon } \\
\text { Adenocarcinoma) }\end{array}$ \\
\hline 32 & $70, \mathrm{~F}$ & Adenocarcinoma & - & 4 & - & IV & Adenocarcinoma \\
\hline 33 & $80, \mathrm{M}$ & Insufficient material & Insufficient material & - & 2 & Ia & Adenocarcinoma* \\
\hline 34 & $83, \mathrm{M}$ & Adenocarcinoma & - & - & 1 & IV & Adenocarcinoma \\
\hline 35 & $72, \mathrm{M}$ & Adenocarcinoma & - & 5 & 10 & pT1bN0Ia & Adenocarcinoma \\
\hline 36 & $83, \mathrm{~F}$ & Adenocarcinoma & - & - & 8 & IIIa & Adenocarcinoma \\
\hline 37 & $75, \mathrm{M}$ & Mtx (Intestinal Adenocarcinoma) & - & 2 & 4 & - & Mtx (Colon Adenocarcinoma) \\
\hline 38 & $74, \mathrm{~F}$ & Adenocarcinoma & - & 4 & 1 & IV & Adenocarcinoma \\
\hline 39 & $63, \mathrm{~F}$ & Adenocarcinoma & - & - & 1 & Ia & Adenocarcinoma \\
\hline 40 & $73, \mathrm{~F}$ & Adenocarcinoma & Adenocarcinoma & 10 & 3 & IV & Adenocarcinoma \\
\hline 41 & $49, \mathrm{~F}$ & Mtx (Breast ca) & - & 5 & 3 & & Mtx (Breast ca) \\
\hline 42 & $81, \mathrm{M}$ & Adenocarcinoma & - & 3 & 1 & Ia & Adenocarcinoma \\
\hline 43 & $75, \mathrm{M}$ & Inflammatory cells & Abscess & - & - & - & Abscess \\
\hline 44 & $69, \mathrm{M}$ & Adenocarcinoma & Adenocarcinoma & - & 3 & Ia & Adenocarcinoma \\
\hline 45 & $51, \mathrm{M}$ & Adenocarcinoma & - & 2 & 1 & Ia & Adenocarcinoma \\
\hline 46 & $70, \mathrm{M}$ & Squamous cell ca & - & - & - & pT2bN0IIa & Squamous cell ca \\
\hline 47 & $71, \mathrm{M}$ & Mtx (neuroendocrine ca) & - & 5 & 3 & - & Mtx (Neuroendocrine ca) \\
\hline 48 & $84, \mathrm{M}$ & Adenocarcinoma & Adenocarcinoma & - & - & IV & Adenocarcinoma \\
\hline 49 & $74, \mathrm{M}$ & Squamous cell ca & - & - & - & IV & Squamous cell ca \\
\hline 50 & $64, \mathrm{M}$ & Insufficient material & - & - & - & - & Amarthocondroma \\
\hline 51 & $74, \mathrm{~F}$ & Squamous cell ca & - & - & - & IIIa & Squamous cell ca \\
\hline 52 & $76, \mathrm{~F}$ & Adenocarcinoma & - & - & - & pT2aNOIIa & Adenocarcinoma \\
\hline 53 & $85, \mathrm{~F}$ & Neuroendocrine malignant & Neuroendocrine malignant & - & - & IV-diffuse & Neuroendocrine malignant \\
\hline 54 & $65, \mathrm{M}$ & Adenocarcinoma & - & - & - & Ia & Adenocarcinoma \\
\hline 55 & $83, \mathrm{M}$ & Squamous cell ca & - & - & - & Ia & Squamous cell ca \\
\hline 56 & $62, \mathrm{~F}$ & Adenocarcinoma & - & - & - & Ia & Adenocarcinoma \\
\hline 57 & $68, \mathrm{~F}$ & Mtx (Breast ca) & - & 2 & 1 & - & Mtx (Breast ca) \\
\hline 58 & $82, \mathrm{~F}$ & Adenocarcinoma & - & - & - & $\mathrm{Ib}$ & Adenocarcinoma \\
\hline 59 & $76, \mathrm{M}$ & Adenocarcinoma & - & - & - & Ia & Adenocarcinoma \\
\hline 60 & $64, \mathrm{~F}$ & Neuroendocrine tumor & - & - & - & pT1aN0Ia & Typical carcinoid \\
\hline 61 & $66, \mathrm{M}$ & Carcinoid & Carcinoid & - & - & pT1aN0Ia & Typical carcinoid \\
\hline 62 & $79, \mathrm{~F}$ & Insufficient material & Insufficient material & 5 & 2 & IV & Adenocarcinoma* \\
\hline 63 & $75, \mathrm{~F}$ & Inconclusive & Inconclusive & 3 & 10 & pT2bN1IIb & Adenocarcinoma \\
\hline 64 & $52, \mathrm{~F}$ & Mtx (Intestinal adenocarcinoma) & - & - & 4 & - & Mtx (Colon Adenocarcinoma) \\
\hline 65 & $75, \mathrm{M}$ & SCLC & - & 6 & 5 & - & SCLC \\
\hline 66 & $83, \mathrm{M}$ & NSCLC & Squamous cell ca & - & - & IIIa & Squamous cell ca \\
\hline 67 & $29, \mathrm{M}$ & Amarthocondroma & Amarthocondroma & - & - & - & Amarthocondroma \\
\hline 68 & $72, \mathrm{~F}$ & Adenocarcinoma & - & - & - & Ia & Adenocarcinoma \\
\hline 69 & $67, \mathrm{M}$ & Mtx (Pancreatic Adenocarcinoma) & - & 2 & 1 & IV & Mtx (Pancreas Adenocarcinoma) \\
\hline 70 & $55, \mathrm{~F}$ & Adenocarcinoma & Adenocarcinoma & - & 5 & $\mathrm{Ib}$ & Adenocarcinoma \\
\hline 71 & $85, \mathrm{~F}$ & Squamous cell ca & Squamous cell ca & - & - & $\mathrm{Ib}$ & Squamous cell ca \\
\hline 72 & $68, \mathrm{~F}$ & Inflammatory cells & Inflammation & - & - & - & Inflammation \\
\hline 73 & $79, \mathrm{M}$ & Mtx (intestinal Adenocarcinoma) & Mtx (intestinal Adenocarcinoma) & - & 3 & - & Mtx (Colon adenocarcinoma) \\
\hline 74 & $75, \mathrm{M}$ & Adenocarcinoma & - & - & 10 & $\mathrm{Ib}$ & Adenocarcinoma \\
\hline 75 & $51, \mathrm{M}$ & Squamous cell ca & Squamous cell ca & - & - & IIa & Squamous cell ca \\
\hline
\end{tabular}

$\mathrm{Ca}=$ carcinoma; FNAB = Fine Needle Aspiration Biopsy; Mtx = metastasis; NSCLC NOS = Non Small Cell Lung Cancer Not Otherwise Specified; SCLC = Small Cell Lung Carcinoma. * Diagnosis made with Trans-Bronchial Needle Aspiration biopsy. 


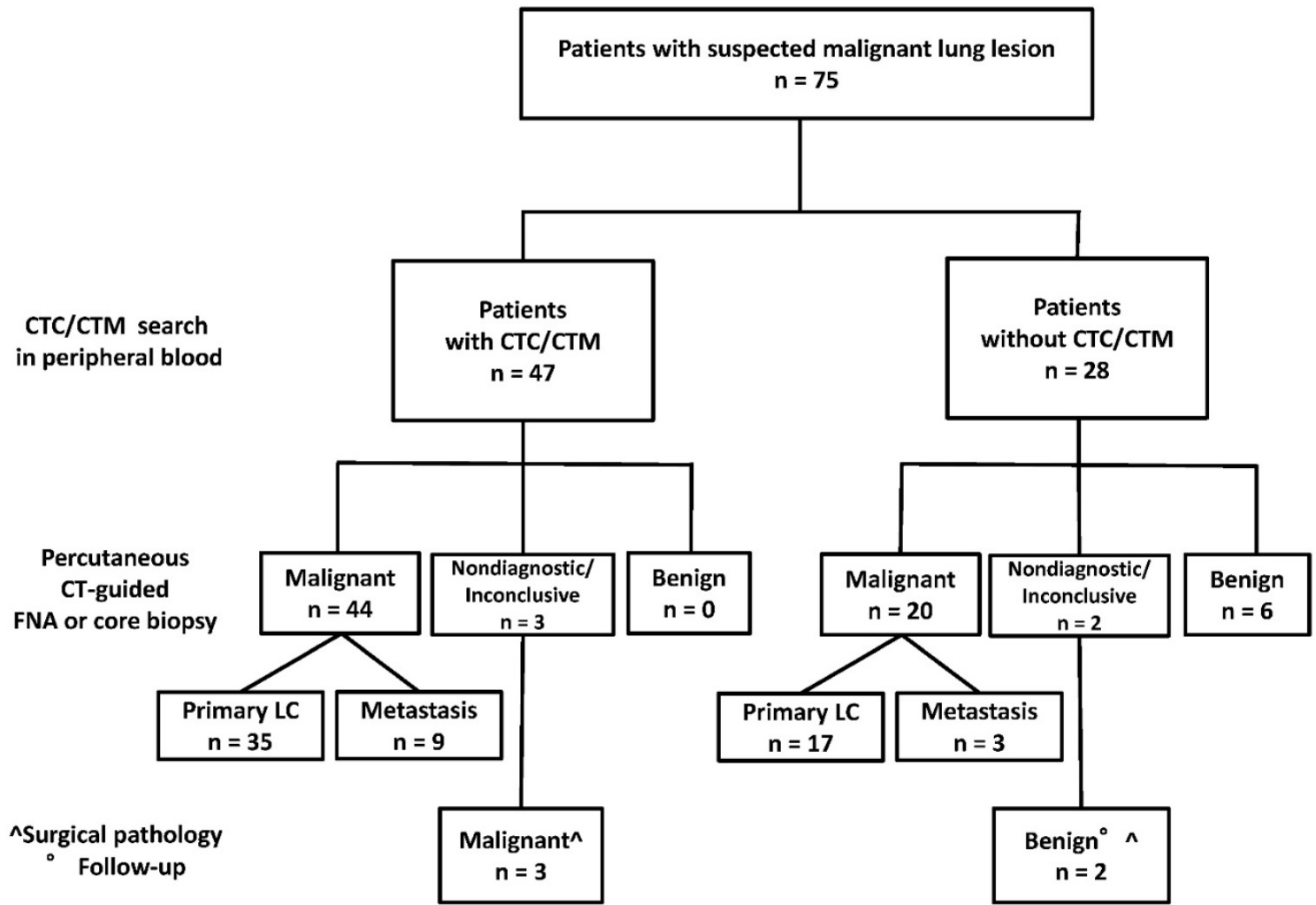

Figure 1: Results of CTC/CTM search and percutaneous FNA or core biopsy in 75 patients.

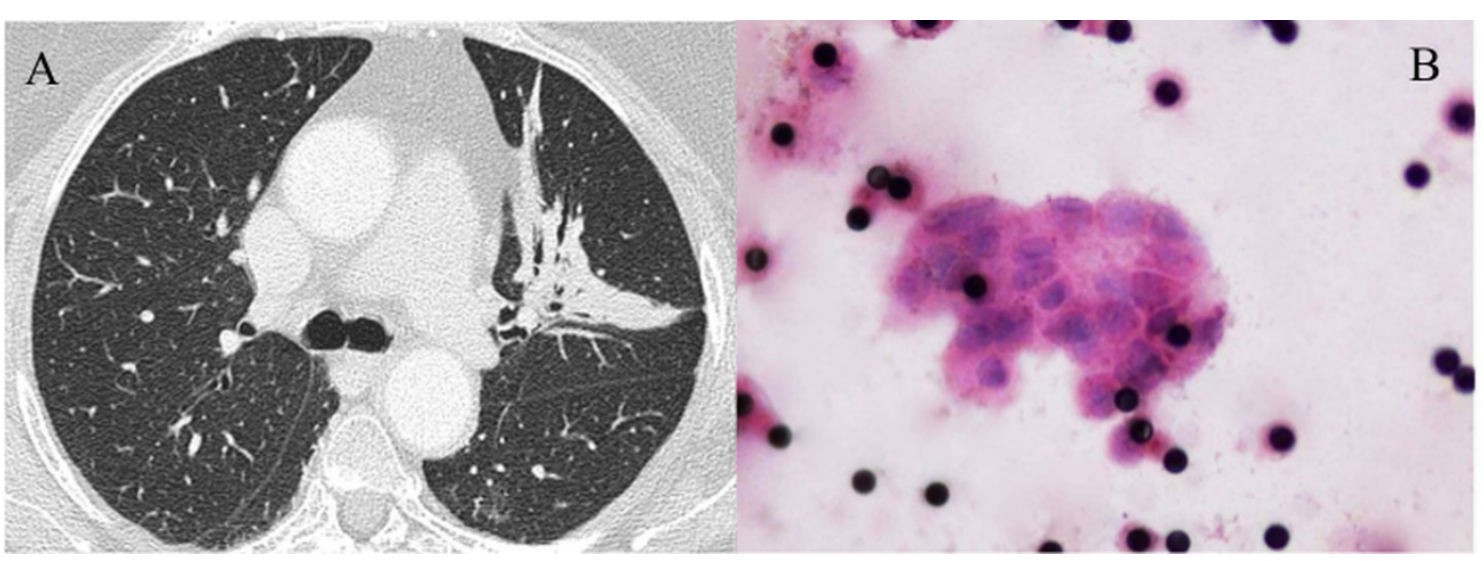

Figure 2 (A-B): CTM in a case (number 63) of inconclusive FNA and core biopsy. (A) CT shows a consolidation in the left upper lobe with air bronchogram and small bronchiectases. (B) Filter (Hematoxylin and Eosin staining, original magnification $\times 1,000$ ) showing a CTM formed by more than 20 CTC with variably increased and irregularly shaped nuclei and multiple $8 \mu \mathrm{m}$ pores of the filter appearing as black dots. Surgical pathology demonstrated a stage Ilb adenocarcinoma.

Overall sensitivity of CTC/CTM presence for malignancy was $70.1 \%$ (95\% CI: 56.9-83.1\%), specificity 100\%, PPV 100\% and NPV 28.6\% (95\%CI: 11.9-45.3\%).

Remarkably, the presence of CTC/CTM anticipated the diagnosis of primary lung cancer in 3 of 5 patients with non-diagnostic or inconclusive results of FNA or core biopsy, whereas CTC/CTM were not observed in 1 patient with sarcoidosis and 1 with amarthocondroma.

CTC/CTM were present in 17 of 29 (58\%) patients with stage I-II and in 21 of $26(80 \%)$ patients with stage III-IV primary lung cancer and in 31 of 38 $(81.5 \%)$ patients with adenocarcinoma and in 5 of 12
(42\%) patients with squamous cell carcinoma.

\section{Discussion}

Percutaneous CT-guided FNA and core biopsy are established diagnostic procedures for patients with peripheral nodule or mass of suspicious malignant nature [19]. The $93.3 \%$ diagnostic yield of FNA (with ROSE) and core biopsy in our series is in line with previous data $[9,19]$.

So far the diagnostic role of CTC/CTM search for lung cancer has been scarcely explored. One study evaluated CTC/CTM search with the ISET filtration method to anticipate lung cancer development in 
patients with chronic obstructive pulmonary disease who were followed up with $\mathrm{CT}$ until lung nodules were detected [20]. This clinical context is different from ours in which we investigated the capability of CTC/CTM to anticipate malignancy in patients with evidence of suspicious lung nodules or masses on CT. In particular while sensitivity is a major concern for studies addressing screening of lung cancer, specificity is the crucial feature requested to a diagnostic tool in patients with high suspicion of lung cancer.

In the present study, we used the ScreenCell Cyto filtration method that allows isolation by cell size and does not involve any biological characterization of the putative CTC to preliminarily explore the predictive value for malignancy of detection of CTC/CTM in a prospective consecutive series of patients undergoing diagnostic FNA or core biopsy of pulmonary lesions of suspected malignant nature. Notably, in all our patients the blood sample for CTC search was taken before the diagnostic FNA or core biopsy by using the venous access that is routinely prepared in order to aid for therapeutic intervention in case of systemic adverse effects during or after the FNA or core biopsy procedure (hypotension, etc.). This time point for blood sampling enables us to get rid of the possibility that manipulation of lung cancer in diagnostic procedures including FNA or core biopsy determines delivery of CTC in the blood stream $[11,12]$.

There is growing evidence that CTM do not derive from CTC conglomeration in the blood stream and rather represent cell aggregates originating in the tumor and entering the vasculature, possibly as the result of the surface expression of specific molecules promoting cellular adhesion [21, 22]. CTM might be more capable of metastasizing as compared with single CTC [22]. Moreover, the presence and number of CTM is a poor prognostic factor, in particular for patients with small cell lung cancer [23, 24]. However, since we were interested here in the potential diagnostic predictive value rather than prognostic role of CTC and CTM, the present study considered their presence both separately and altogether.

In prior studies evaluating possible contribute of presence of CTC or CTM in cases with putative malignant lesions of solid primary tumors of pancreas, stomach, breast and prostate, the overall sensitivity for malignant lesions ranged between 42 and $86 \%$ [5-8], whereas specificity was higher and in the $91-100 \%$ range [5-8]. Using antibodies for detection of CTC (Veridex ${ }^{\circledR}$ LLC; NJ, USA), Maestro et al. [25] found 2 or more CTCs $/ 7.5 \mathrm{~mL}$ in $31.5 \%$ of 438 patients with several types of primary cancer (breast, colorectal, prostate) but in none of 106 healthy control subjects. The $62.3 \%$ of patients with metastatic disease had 2 or more CTCs/7.5 mL as compared to only $14.0 \%$ of those with localized disease. The presence of CTCs was correlated to stage in the three studied tumor types and no differences in the number of cells were found between them.

When we considered the whole series of our patients we obtained a sensitivity of $70 \%$ and specificity of $100 \%$. Sensitivity was $69 \%$ in primary lung cancers $(58 \%$ in stage I-II lesions and $80 \%$ in stage III-IV lesions) and $75 \%$ in metastases.

The $69 \%$ positivity of CTC/CTM in our series of 55 primary lung cancers of different type and stage is substantially in line with the $46-80 \%$ CTC prevalence reported in previous series of early or advanced non small cell lung cancer [14, 16, 17, 26, 27].

Sensitivity, specificity and positive and negative predictive values in our series indicate that CTC/CTM presence in peripheral blood samples may be a highly specific but rather insensitive marker of the malignant nature of pulmonary lesions scheduled for percutaneous CT-guided biopsy. The reasons why CTC/CTM presence is detectable in most but not all cases of lung cancers and metastases, implying low sensitivity of the method [28], are not established. CTC/CTM detection may be influenced by several factors, including performance of the detection method, intermittent blood release of CTC from the primary tumor, and a lymphatic rather than blood tumor cell release.

Lack of CTC/CTM in all our 8 patients with FNA or core biopsy diagnosed benign pathology may be of interest. In fact, although CTC or CTM usually do not occur in healthy subjects [25, 29], our data indicate a potential role of CTC search in the clinical setting. Circulating non-hematological cells (CNHCs) which can be misdiagnosed as CTC have been reported in patients without cancers, namely with benign thyroid, parathyroid and pancreas pathologies $[29,30]$. However, application of cytomorphological criteria developed to characterize the malignant or non-malignant nature of CNHCs can solve the issue [4]. Obviously, data in larger cohorts of patients with benign pulmonary lesions are required to further assess specificity of CTC/CTM presence.

In our opinion, also the data we obtained in the 5 patients who had non-diagnostic or inconclusive results of percutaneous FNA or core biopsy are of note. In fact, they suggest that CTC/CTM presence can anticipate malignancy and may theoretically represent a useful clue in cases with non-diagnostic or inconclusive FNA or core biopsy.

We recognize the following limitations of our study. First, CTC/CTM detection based on cytomorphometric criteria entails some operator 
dependence. However, the reported inter-operator variability for CTC identification using the cytomorphometric criteria we adopted is low [16] and filter visual assessment was performed in consensus by the cytopathologists. Second, CTC or CTM detection per se is certainly not sufficient for diagnostic purposes. In particular we did not address the capability of CTC/CTM characterization to differentiate between primary lung cancer and metastatic lung nodules from extra-pulmonary tissues, which is fundamental for diagnostic purposes. From this point of view, as immunocytochemistry in the FNA or core biopsy is capable to establish the primary or secondary nature of the lung malignant lesion, it can be anticipated that a similar phenotypic characterization of CTC/CTM with specific markers may overcome this limitation. Immunocytochemistry characterization of the CTC and CTM isolated with filtration is possible and represents a rapidly growing field $[4,7,8,31-34]$, but it was not performed here. Alternatively, genomic and expression analysis of CTC can be useful for such a purpose [2].

In conclusion, our preliminary data suggest that presently, due to the low sensitivity, the search of CTC/CTM cannot replace CT guided percutaneous FNA or core biopsy in the diagnostic work-up of patients with suspicious malignant lung lesions. However, the high specificity may as yet indicate a role in cases with non-diagnostic or inconclusive FNA or core biopsy results to be further investigated.

\section{Acknowledgments}

This work was supported by Ente Cassa di Risparmio di Firenze and Regione Toscana ("CYTOPEM" POR CREO FSE 2007-2013), by Associazione Italiana per la Ricerca sul Cancro (IG-2015 17691), and by EC H2020 FRAMEWORK PROGRAMME (Project's title: SPIDIA4P: SPIDIA for Personalized Medicine - Standardisation of generic Pre-analytical procedures for In-vitro DIAgnostics for Personalized Medicine-GA n 733112).

\section{Competing Interests}

The authors have declared that no competing interest exists.

\section{References}

1. Ignatiadis M, Lee M, Jeffrey SS. Circulating tumor cells and circulating tumor DNA: challenges and opportunities on the path to clinical utility. Clin Cancer Res. 2015;21:4786-800.

2. Ilie M, Hofman V, Long E, et al. Current challenges for detection of circulating tumor cells and cell-free circulating nucleic acids, and their characterization in non-small cell lung carcinoma patients. What is the best blood substrate for personalized medicine? Ann Transl Med. 2014;2:107.

3. Zhang Z, Ramnath N, Nagrath S. Current status of CTCs as liquid biopsy in lung cancer and future directions. Front Oncol. 2015;5:209.
4. Hofman VJ, Ilie M, Hofman PH. Detection and characterization of circulating tumor cells in lung cancer: Why and how? Cancer Cytopthol.2015; doi: 10.1002/cncy.21651

5. Iwanicki-Caron I, Basile P, Toure E, et al. Usefulness of circulating tumor cell detection in pancreatic adenocarcinoma diagnosis. Am J Gastroenterol. 2013;108:152-5.

6. Tang L, Zhao S, Liu W, et al. Diagnostic accuracy of circulating tumor cells detection in gastric cancer: systematic review and meta-analysis. BMC Cancer. 2013;13:314

7. Murray NP, Miranda R, Ruiz A, Droguett E. Diagnostic yield of primary circulating tumor cells in women suspected of breast cancer: the BEST (Breast Early Screening Test) study. Asian Pac J Cancer Prev. 2015;16:1929-34.

8. Murray NP, Reyes E, Tapia P, et al. Diagnostic performance of malignant prostatic cells detection in blood for early detection of prostate cancer: comparison to prostatic biopsy. Arch Esp Urol. 2011;64:961-71.

9. Mazza E, Maddau C, Ricciardi A, Falchini M, Matucci M, Ciarpallini T. On-site evaluation of percutaneous CT-guided fine needle aspiration of pulmonary lesions. A study of 321 cases. Radiol Med. 2005;110:141-8.

10. Travis WD, Brambilla E, Nicholson AG, Yatabe Y, Austin JHM. The 2015 World Health Organization classification of lung tumors. Impact of genetic, clinical and radiologic advances since the 2004 classification. J Thorac Oncol. 2015;10:1243-60.

11. Sawabata N, Okumura M, Utsumi T, et al. Circulating tumor cells in peripheral blood caused by surgical manipulation of non-small-cell lung cancer: pilot study using an immunocytology method. Gen Thorac Cardiovasc Surg. 2007;55:189-92.

12. Yao X, Williamson C, Adalsteinsson VA, et al. Tumor cells are dislodged into the pulmonary vein during lobectomy. J Thorac Cardiovasc Surg. 2014;148:3224-31.

13. Travis WD, Brambilla E, Noguchi M, et al. Diagnosis of lung cancer in small biopsies and cytology: implications of the 2011 International Association for the Study of Lung Cancer/American Thoracic Society/European Respiratory Society Classification. Arch Pathol Lab Med. 2013;1375: 668-84.

14. Mascalchi M, Falchini M, Maddau C, et al. Prevalence and number of circulating tumour cells and microemboli at diagnosis of advanced NSCLC. J Cancer Res Clin Oncol. 2016;142:195-200.

15. De Giorgi V, Pinzani P, Salvianti F, et al. Application of a filtration- and isolation-by-size technique for the detection of circulating tumor cells in cutaneous melanoma. J Invest Dermatol 2010;130:2440-7.

16. Hofman VJ, Long E, Ilie M, et al. Morphological analysis of circulating tumour cells in patients undergoing surgery for non-small cell lung carcinoma using the isolation by size of epithelial tumour cell (ISET) method. Cytopathology 2012;23:30-8.

17. Krebs MG, Hou JM, Sloane R, et al. Analysis of circulating tumor cells in patients with non-small cell lung cancer using epithelial marker-dependent and -independent approaches. J Thorac Oncol. 2012;7:306-15.

18. Detterbeck FC, Boffa DJ, Tanoue LT. The new lung cancer staging system. Chest. 2009; 136:260-271.

19. Wahidi MM, Govert JA, Goudar RK, et al. Evidence for the treatment of patients with pulmonary nodules: when is it lung cancer? ACCP evidence-based clinical practice guidelines (2nd edition). Chest. 2007;132(3 Suppl):94S-107S.

20. Ilie M, Hofman V, Long-Mira E, et al. "Sentinel" circulating tumor cells allow early diagnosis of lung cancer in patients with chronic obstructive pulmonary disease. PLoS ONE 2014; 9(10): e111597. doi:10.1371/journal.pone.0111597

21. Aceto N, Bardia A, Miyamoto DT, et al. Circulating tumor cell clusters are oligoclonal precursors of breast cancer metastasis. Cell. 2014;158:1110-22

22. Bottos A, Hynes N. Staying together on the road to metastasis. Nature 2014;514:309-10.

23. Hou JM, Krebs MG, Lancashire L, et al. Clinical significance and molecular characteristics of circulating tumor cells and circulating tumor microemboli in patients with small-cell lung cancer. J Clin Oncol. 2012; 30:525-32.

24. Crosbie PA, Shah R, Krysiak P, et al. Circulating tumor cells detected in the tumor-draining pulmonary vein are associated with disease recurrence after surgical resection of NSCLC. J Thorac Oncol. 2016;11:1793-7.

25. Maestro LM, Sastre J, Rafael SB, et al. Circulating tumor cells in solid tumor in metastatic and localized stages. Anticancer Res. 2009; 29:4839-43.

26. Wendel M, Bazhenova L, Boshuizen R, et al. Fluid biopsy for circulating tumor cell identification in patients with early and late stage non-small cell lung cancer; a glimpse into lung cancer biology. Phys Biol. 2012;9:016005. 
27. Carlsson A, Nair VS, Luttgen MS, et al. Circulating tumor microemboli diagnostics for patients with non-small-cell lung cancer. J Thorac Oncol.2014;9:1111-9.

28. Wicha MS, Hayes DF. Circulating tumor cells: not all detected cells are bad and not all bad cells are detected. J Clin Oncol. 2011;29:1508-11.

29. Hofman VJ, Ilie MI, Bonnetaud C, et al. Cytopathologic detection of circulating tumor cells using the isolation by size of epithelial tumor cell method: promises and pitfalls. Am J Clin Pathol. 2011;135:146-56.

30. Cauley CE, Pitman MB, Zhou J, et al. Circulating epithelial cells in patients with pancreatic lesions: clinical and pathologic findings. J Am Coll Surg. 2015;22:699-707.

31. Lecharpentier A, Vielh P, Perez-Moreno P, et al. Detection of circulating tumour cells with a hybrid (epithelial/mesenchymal) phenotype in patients with metastatic non-small cell lung cancer. $\mathrm{Br} \mathrm{J}$ Cancer. 2011;105:1338-41.

32. Hou JM, Krebs M, Ward T, et al. Circulating tumor cells as a window on metastasis biology in lung cancer. Am J Pathol. 2011;178:989-96.

33. Park SM, Wong DJ, Ooi CC et al. Molecular profiling of single circulating tumor cells from lung cancer patients. Proc Natl Acad Sci U S A. 2016;113:E8379-86.

34. Dhar M, Pao E, Renier C et al. Label-free enumeration, collection and downstream cytological and cytogenetic analysis of circulating tumor cells. Sci Rep. 2016; 6:35474 doi: 10.1038/srep35474. 